Review

\title{
Distributed Discrimination of Strain and Temperature Based on Brillouin Dynamic Grating in an Optical Fiber
}

\author{
Kazuo HOTATE ${ }^{1 *}$, Weiwen ZOU², Rodrigo Kendy YAMASHITA ${ }^{1}$, and Zuyuan HE$^{2}$ \\ ${ }^{1}$ Department of Electrical Engineering and Information Systems, The University of Tokyo, Japan \\ ${ }^{2}$ State Key Laboratory Advanced Optical Communication Systems and Networks, Department of Electronic Engineering, \\ Shanghai Jiao Tong University, China \\ "Corresponding author: Kazuo HOTATE＿E-mail: hotate@sagnac.t.u-tokyo.ac.jp
}

\begin{abstract}
This paper reviews distributed discrimination of strain and temperature by use of an optical fiber based on fiber optic nerve systems. The preliminary method based on multiple resonance peaks of the Brillouin gain spectrum in a specially-designed fiber is firstly introduced. The complete discrimination of strain and temperature based on the Brillouin dynamic grating in a polarization maintaining fiber is extensively presented. The basic principle and two experimental schemes of distributed discrimination based on fiber optic nerve systems are demonstrated. The performance of the high discriminative accuracy $\left(0.1{ }^{\circ} \mathrm{C}-0.3{ }^{\circ} \mathrm{C}\right.$ and $\left.5 \mu \varepsilon-12 \mu \varepsilon\right)$ and high spatial resolution $(\sim 10 \mathrm{~cm})$ with the effective measurement points of about 50 for a standard system configuration or about 1000 for a modified one will be highly expected in real industry applications.
\end{abstract}

Keywords: Fiber optic nerve systems, Brillouin optical correlation-domain analysis, Brillouin dynamic grating, distributed discrimination, strain, temperature

Citation: Kazuo HOTATE, Weiwen ZOU, Rodrigo Kendy YAMASHITA, and Zuyuan HE, "Distributed Discrimination of Strain and Temperature Based on Brillouin Dynamic Grating in an Optical Fiber," Photonic Sensors, DOI: 10.1007/s13320-013-0130-7.

\section{Introduction}

The fiber optic nerve system of the Brillouin optical correlation-domain analysis (BOCDA) [1-3] or reflectometry (BOCDR) [4-6] takes use of the correlation-domain continuous-wave technique, which is more advantageous than the Brillouin optical time-domain analysis (BOTDA) [7,8] and reflectometry (BOTDR) [9, 10]. Fiber optic nerve systems (BOCDA and BOCDR) have been theoretically investigated and experimentally realized to provide outstanding performances in diagnosis of fully-distributed strain or temperature disturbances with an extremely-high spatial resolution of from centimeters $[1,2,4,5]$ to several millimeters $[3,6]$ along the whole fiber under test
(FUT). Regardless of the ability of how short segment can be diagnosed, either BOCDA/R or BOTDA $/ \mathrm{R}$ via measurement of a single parameter (i.e., Brillouin frequency shift, BFS) is unable to distinguish the response to strain from the response to temperature [11-13].

In this paper, we demonstrate the research trends of distributed discrimination of strain and temperature by use of an optical fiber based on fiber optic nerve systems. Firstly, the preliminary method based on multiple acoustic modes in a specially designed fiber is introduced. Secondly, the principle and experimental demonstration of the complete discrimination of strain and temperature based on the Brillouin dynamic grating (BDG) in a polarization maintaining are presented. Finally, the

Received: 16 July 2013 / Revised version: 30 August 2013

(C) The Author(s) 2013. This article is published with open access at Springerlink.com 
development of distributed discrimination of strain and temperature is reviewed.

\section{Preliminary method}

\subsection{Mechanism of the Brillouin-based sensing technique}

Brillouin scattering is a photon-phonon interaction similar to the case of Raman scattering [14]. Brillouin scattering is due to the fact that annihilation of a pump photon creates a Stokes photon and a phonon simultaneously. The phonon is the vibrational modes of atoms, which is also called a propagation density wave or an acoustic phonon. The newly-generated photon suffers a down-shifted frequency from the pump photon due to the Doppler shift associated with the fact that the generated acoustic phonons move forward. The down-shifted frequency is called the Brillouin frequency shift $\left(\mathrm{BFS}, v_{B}\right)$ given by

$$
v_{B}=\frac{2 n_{\text {eff }}}{\lambda} \cdot V_{a}
$$

where $\lambda$ is the optical wavelength, $n_{\text {eff }}$ the effective refractive index, and $V_{a}$ the effective acoustic velocity [14]. The BFS in silica optical fibers is $10 \mathrm{GHz}-11 \mathrm{GHz}$ in the $1550-\mathrm{nm}$ region, as shown in Fig. 1 [13].

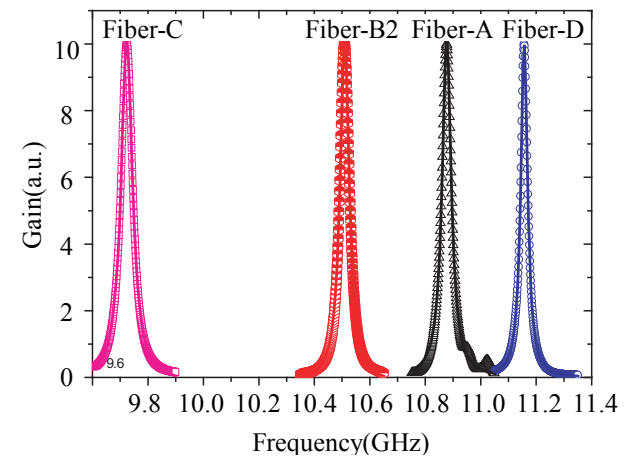

Fig. 1 Measured (symbolic points) and Lorenzian fitted (solid curves) BGS of Fiber-A, Fiber-B2, Fiber-C, and Fiber-D with different dopant concentrations and different-diameter cores [13] (copyright @ JLT).

As depicted in Fig.2, it is clarified that the BFS change $\left(\delta v_{B}\right)$ has a good linear dependence on strain $(\delta \varepsilon)$ and/or temperature $(\delta T)$ [11-13], which can be expressed by

$$
\delta v_{B}=C_{v}^{\varepsilon} \cdot \delta \varepsilon+C_{v}^{T} \cdot \delta T
$$

where $C_{v}{ }^{\varepsilon}(=0.04 \mathrm{MHz} / \mu \varepsilon-0.05 \mathrm{MHz} / \mu \varepsilon)$ and $C_{v}{ }^{T}$ $\left(=0.9 \mathrm{MHz} /{ }^{\circ} \mathrm{C}-1.1 \mathrm{MHz} /{ }^{\circ} \mathrm{C}\right)$ denote the strain and temperature coefficients. The relation given in (2) is the basic mechanism of Brillouin distributed sensing techniques [1-10], however, gives a challenge how to discriminate their responses by use of a single-length optical fiber.
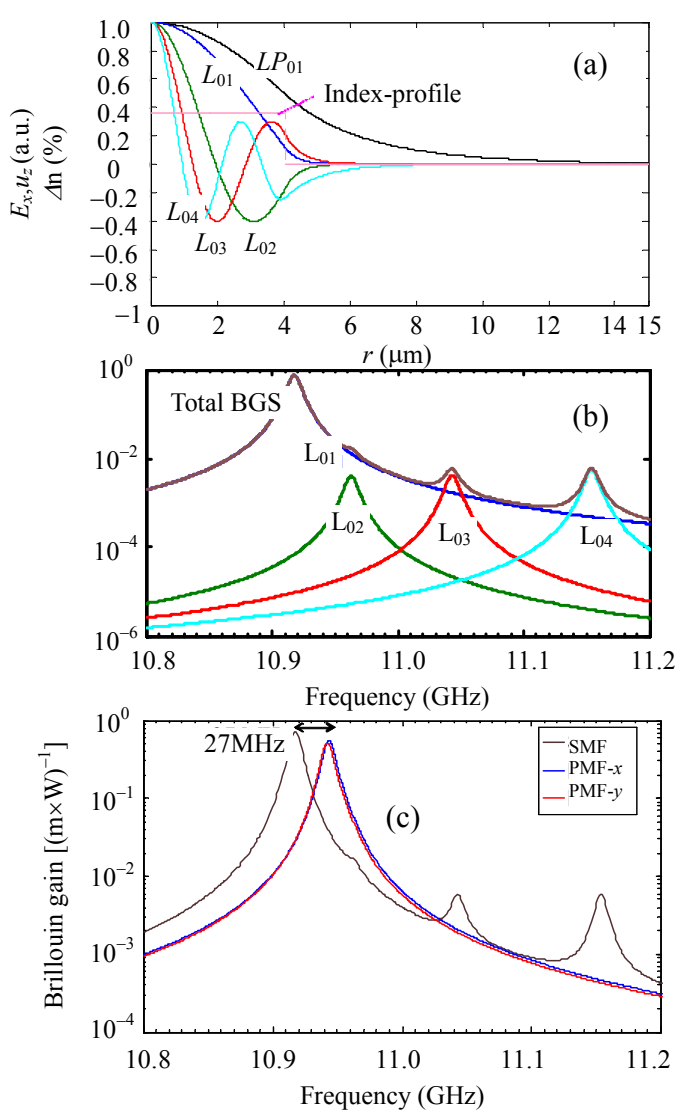

Fig. 2 Simulation results: (a) evaluated field distributions of the optical $\mathrm{LP}_{01}$ mode and four longitudinal acoustic $L_{01}$ modes in the SMF, (b) total BGS (solid curve) composed of four $L_{01}$ modes induced the BGS (dashed curves), and (c) experimentally measured result [18] (copyright @ IEEE PTL).

\subsection{Multiple peaks in a specially-designed optical fiber}

A preliminary method is to use a specially-designed fiber with multiple Brillouin peaks that exhibit different frequency variations in temperature and strain [15-17]. As shown in Fig. 2 
[18], there are four resonance peaks of the Brillouin gain spectrum (BGS) even in a standard single mode optical fiber (SMF). This is because the acoustic wavenumber is far larger than the optical one [19] so that four acoustic modes can simultaneously exist in an SMF.

The BGS in a w-shaped high-delta optical fiber with the F-doped depressed inner cladding (F-HDF) has four well-separated peaks, as shown in Fig. 3 [17]. The first-order and the fourth-order (see Fig. 4) are more suitable for the discrimination of strain and temperature since they are attributed to two separate layers of the $\mathrm{GeO}_{2}$-doped core and F-doped inner cladding [19-21].

Although the preliminary method based on a specially-designed fiber [15-17] is a frequencybased measurement technique prior to the power-based one [22], it is still ill-conditioned and physically limited due to the correlated relationship among multiple peaks

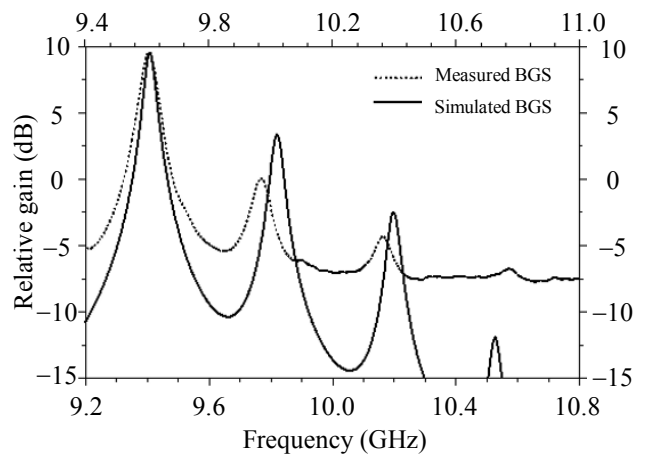

Fig. 3 Typical BGS measured at $25{ }^{\circ} \mathrm{C}$ in the loose state (dotted curve) compared with the simulated BGS (solid curve) in the F-HDF: bottom axis - measured BGS; top axis simulated BGS [17] (copyright @ OSA OL).

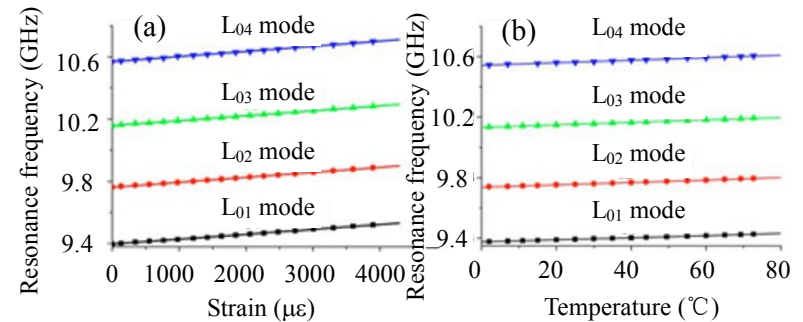

Fig. 4 Resonance frequencies of different acoustic modes as a function of (a) strain and (b) temperature: solid lines least-squares linearly fits to data. [17] (copyright @ OSA OL).

\section{Complete discrimination of strain and temperature}

\subsection{Principle}

A Panda-type polarization-maintaining fiber (PMF), which has been widely used for optical fiber communications [23] or fiber-optic sensors [24], is composed of two $\mathrm{B}_{2} \mathrm{O}_{3}$-doped-silica stress-applying parts that are inserted in a pure-silica cladding and symmetrically placed beside a $\mathrm{GeO}_{2}$-doped-silica core [see Figs. 5(a) and 5(b)]. Due to the difference in thermal contraction between $\mathrm{B}_{2} \mathrm{O}_{3}$-doped silica and pure silica, two-dimensional stress is raised and stored into the core during the drawing process in fiber fabrication [23]. The residual stress makes the refractive index along $x$-axis $\left(n_{x}\right)$ slightly greater than that along $y$-axis $\left(n_{y}\right)$. Consequently, a lightwave linearly polarized along $x$-axis (so called slow axis) propagates slower than that along $y$-axis (fast axis). The difference between $n_{x}$ and $n_{y}$ (i.e., birefringence $B=n_{x}-n_{y}$ ) is small (e.g., $B=$ $\sim 3.3 \times 10^{-4}$ for the fiber used here) but large enough so that lightwaves linearly-polarized along either $x$ or $y$-axis can propagate through the fiber while maintaining their polarization states in despite of the external disturbance.

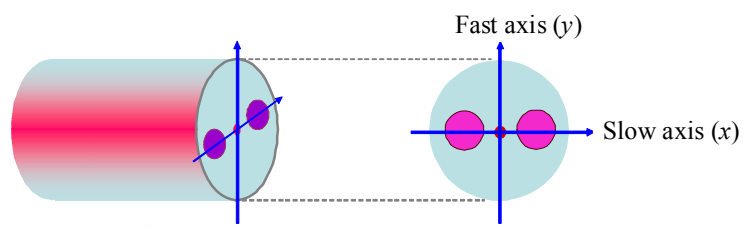

(a)

(b)

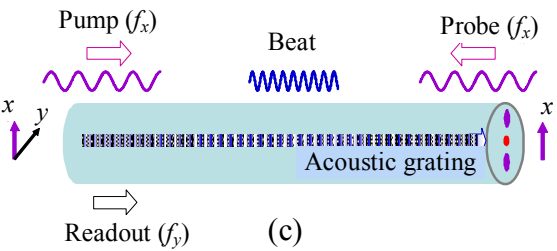

Fig. 5 Schematic diagram of the Panda-type PMF: (a) side view, (b) cross section, and (c) principle of the BDG generation and readout.

As schematically shown in Fig. 5(c), two lightwaves, one is called pump with the optical frequency $f_{x}$ and the other called probe with the 
frequency $f_{x}^{\prime}$, counter-propagate through the fiber; the pump and the probe are linearly polarized along $x$-axis, for instance. When the frequency difference between the pump and probe $f_{x}-f_{x}$ ' equals the BFS given by (1), SBS occurs in which an acoustic wave (phonons) propagating at the velocity $V_{a}$ is significantly generated. The acoustic wave stretches or compresses the fiber core longitudinally and thus modulates periodically the refractive index. Consequently, a Bragg grating moving forward at $V_{a}$ is formed. Because the acoustic wave as a longitudinal wave exhibits no dependence on transverse polarization [18], the $y$-polarized readout light can also be strongly diffracted by the acoustic grating generated by the $x$-polarized pump and probe, as long as the frequency $f_{y}$ of the $y$-polarized readout light satisfies a phase matching condition that requires $f_{y}$ to deviate from that of the $x$-polarized pump $\left(f_{x}\right)$ by a birefringence-determined frequency deviation $[25,26]$

$$
f_{y x}=f_{y}-f_{x}=f_{x} \cdot B / n_{x} .
$$

The residual tensile stress $\left(\sigma_{x y}\right)$ determining the Panda-type PMF's birefringence changes with the ambient temperature $\left(T_{i}\right)$ :

$$
B \propto \sigma_{x y}=k \cdot\left(\alpha_{3}-\alpha_{2}\right) \cdot\left(T_{\mathrm{fic}}-T_{i}\right)
$$

where $T_{\text {fic }}$ denotes the fictive temperature (e.g., $\left.850{ }^{\circ} \mathrm{C}\right)$ of silica glass, $\alpha_{3}\left(\alpha_{2}\right)$ is the thermal coefficient of $\mathrm{B}_{2} \mathrm{O}_{3}$-doped-silica stress-applying parts (pure-silica cladding), and $k$ is a constant determined by the geometrical location of stress-applying parts in the fiber [27]. When temperature increases $\left(\Delta T=T_{i}-25>0\right)$, the residual stress is released, and thus the birefringence decreases as

$$
\Delta B^{T}=-B_{0} \cdot \frac{\Delta T}{T_{\text {fic }}-25}
$$

where $B_{0}$ is the intrinsic birefringence at room temperature $\left(T_{i}=25^{\circ} \mathrm{C}\right)$. In contrast, when an axial strain $\Delta \varepsilon$ is applied to the fiber, additional stress is generated because the stress-applying parts and the cladding contract in the lateral direction differently due to their different Poisson's ratios $\left(\gamma_{3}>\gamma_{2}\right)$ [27], and the birefringence is enlarged with applied strain as

$$
\Delta B^{\varepsilon}=+B_{0} \cdot \frac{\left(\gamma_{3}-\gamma_{2}\right)}{\left(\alpha_{3}-\alpha_{2}\right)\left(T_{\text {fic }}-25\right)} \cdot \Delta \varepsilon .
$$

Consequently, the birefringence-determined frequency deviation $f_{y x}$ varies linearly with a temperature increase and the applied strain, i.e.,

$$
\Delta f_{y x}=f_{y x}-f_{y x 0}=C_{f}^{\varepsilon} \Delta \varepsilon+C_{f}^{T} \Delta T
$$

where $f_{y x 0}$ is the frequency deviation [see (3)] at $25^{\circ} \mathrm{C}$ and in the loose condition, and $C_{f}^{E}$ and $C_{f}^{T}$ are the strain coefficient and the temperature coefficient of the frequency deviation, respectively. According to (4)-(6), one can deduce the strain coefficient

$$
C_{f}^{\varepsilon}=+f_{y x 0} \cdot \frac{\left(\gamma_{3}-\gamma_{2}\right)}{\left(\alpha_{3}-\alpha_{2}\right)\left(T_{\text {fic }}-25\right)}
$$

and temperature coefficient

$$
C_{f}^{T}=-f_{y x 0} \cdot \frac{1}{\left(T_{\text {fic }}-25\right)} .
$$

According to (8) and (9), one knows that the strain coefficient $C_{f}^{\varepsilon}$ is positive while the temperature coefficient $C_{f}^{T}$ is negative, which means that the responses of the birefringence-determined frequency deviation to strain and temperature are in opposite directions. In contrast, $C_{v}^{\varepsilon}$ and $C_{v}^{T}$ in (2) are both positive values because the Young's modulus of silica is nonlinearly increased by strain and temperature [13].

By solving (2) and (7) jointly, we get the strain $(\Delta \varepsilon)$ and temperature $(\Delta T)$ as [26]

$$
\left(\begin{array}{c}
\Delta \varepsilon \\
\Delta T
\end{array}\right)=\frac{1}{C_{v}^{\varepsilon} \cdot C_{f}^{T}-C_{v}^{T} \cdot C_{f}^{\varepsilon}}\left(\begin{array}{cc}
C_{f}^{T} & -C_{v}^{T} \\
-C_{f}^{\varepsilon} & C_{v}^{\varepsilon}
\end{array}\right)\left(\begin{array}{l}
\Delta v_{B} \\
\Delta f_{y x}
\end{array}\right)
$$

In physics, the two phenomena/quantities, i.e., the BFS [ (2)] and the birefringence of the fiber [see (7)], are inherently independent. In mathematics, $C_{f}^{T}$ has a sign opposite to those of other three coefficients, so that the denominator $\left(C_{v}^{\varepsilon} \cdot C_{f}^{T}\right.$ $\left.-C_{v}^{T} \cdot C_{f}^{\varepsilon}\right)$ in (10) has the significant value. Therefore, a complete discrimination of strain and temperature based on simultaneous measurement of the two quantities can be ensured. 


\subsection{Experimental demonstration}

The experimental setup is shown in Fig. 6. Part A in Fig. 6 is a typical pump-probe scheme to measure the BGS (and thus BFS $v_{B}$ ) [7], except that a set of polarization-maintaining (PM) components (polarizer, $\mathrm{PM}$ isolator, $\mathrm{PM}$ circulator, and polarization beam splitter/combiner) are introduced to ensure the pump and probe lightwaves polarized along $x$-axis. The light source is a $1549-\mathrm{nm}$ distributed-feedback laser diode (DFB-LD1). The intensities of the pump and the probe incident to a 31-meter-length fiber under test are amplified with erbium-doped fiber amplifiers to about $140 \mathrm{~mW}$ and about $1 \mathrm{~mW}$, respectively. The frequency of the probe is down-shifted from that of the pump for $10.8 \mathrm{GHz}$ to $11.1 \mathrm{GHz}$ with a single-sideband electro-optic modulator (SSBM) controlled by a microwave synthesizer, so the BGS is obtained as shown in Fig. 7(a), which depicts the BGS of the 31-meter-length FUT. The synchronous detection scheme was employed by chopping the pump with the electro-optic modulator (EOM) and detecting with a lock-in amplifier (LIA1) to ensure high precision in characterization of the BFS $v_{B}$. A reproducible accuracy of $0.1 \mathrm{MHz}$ was confirmed [17].

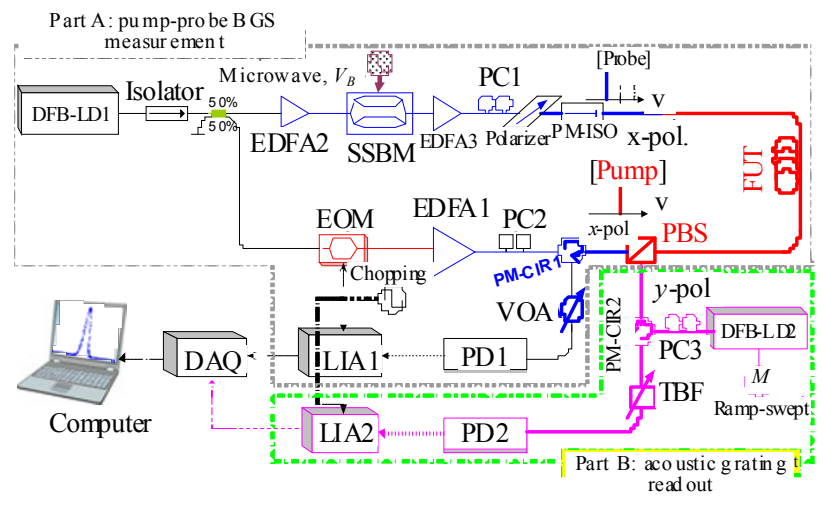

Fig. 6 Configuration of the measurement system: Part A pump-probe scheme to measure the Brillouin gain spectrum and thus the Brillouin frequency shift along $x$-axis; Part B detection of the diffraction spectrum of the acoustic grating in SBS to $y$-polarized readout light [26] (copyright@OSA OE).

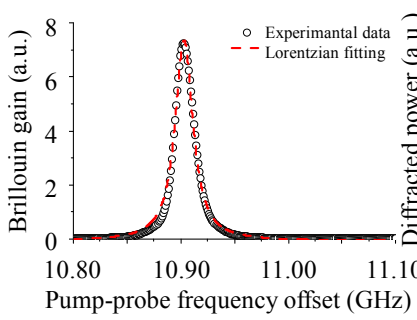

(a)

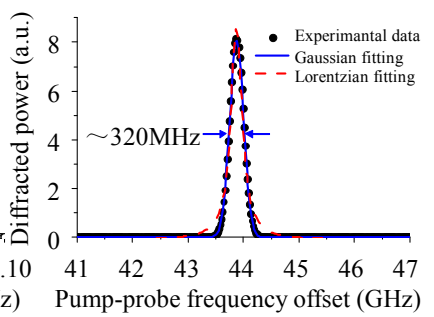

(b)
Fig. 7 Measured Brillouin gain spectrum (a) and the diffraction spectrum of the dynamic acoustic grating induced by SBS to $y$-polarized readout light (b) in a 31-meter-length fiber at room temperature and in the loose condition: circles denote experimental data, and the solid (dashed) curve corresponds to Gaussian (Lorentzian) fitting to experimental data [26] (copyright@OSA OE).

Part B in Fig. 6 is for precisely measuring the BDG's diffraction spectrum to $y$-polarized readout light (and thus the birefringence-determined frequency deviation $f_{y x}$ ). The readout light is generated from the other laser diode DFB-LD2 (central wavelength: $\sim 1549 \mathrm{~nm}$ ), whose optical frequency is ramp-swept by linearly modulating its $\mathrm{dc}$ injection current. Through the polarization beam splitter/combiner after a PM circulator, the linearly polarized readout light (intensity: $\sim 63 \mathrm{~mW}$ ) is launched into the FUT with its polarization along the fiber's $y$-axis. At first, the acoustic phonons in SBS (and thus the dynamic acoustic grating) are maximized by fixing the microwave frequency to the single-sideband modulator at the BFS $v_{B}$ obtained above. Under this condition, the readout light is strongly diffracted by the acoustic grating. The diffracted light is detected synchronously by using the other lock-in amplifier (LIA2) and recorded as a function of the ramp-swept optical frequency of DFB-LD2. In this way, the diffraction spectrum of the acoustic grating to $y$-polarized readout light is obtained. For measuring the diffraction spectrum, a tunable optical band-pass filter with the about $20-\mathrm{GHz}$ bandwidth is used to eliminate the leakage of the $x$-polarized probe and pump. The diffraction spectrum of the dynamic acoustic grating established over the 
31-meter-length FUT measured at room temperature is plotted in Fig. 7(b), showing that the diffraction spectrum has a profile Gaussian-like rather than Lorentzian. By least-squares Gaussian fitting to the experimental data in Fig. 7(b), we get the birefringence-determined frequency deviation $f_{y x}=$ $43.8765 \mathrm{GHz}$ representing the fiber's birefringence $B=3.27773 \times 10^{-4}$, and the full width at the half magnitude (FWHM) as about $320 \mathrm{MHz}$. The repeatability test result validates that the frequency deviation can be measured within a standard error of $\delta f_{y x}=4 \mathrm{MHz}$ corresponding to a reproducible $\delta B=$ $3 \times 10^{-8}$. Comparably, a simply switching the SBS measurement along $x$-axis and $y$-axis [28] can only give the birefringence accuracy around $10^{-5}$, which is insufficient for characterizing strain and temperature responses of the birefringence and for strain and temperature discrimination.

The BGS and the BDG's diffraction spectrum were measured and used to evaluate the $v_{B}$ and $f_{y x}$ when different strains were applied to the 31-meter-length FUT. As summarized in Fig. 8, the

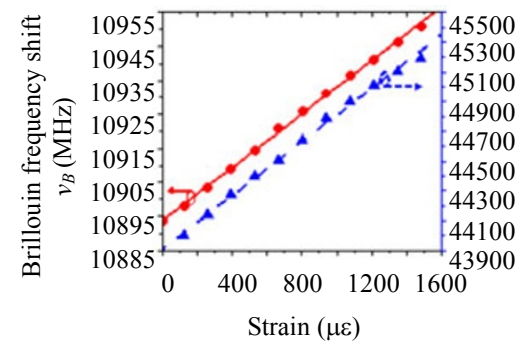

(a)

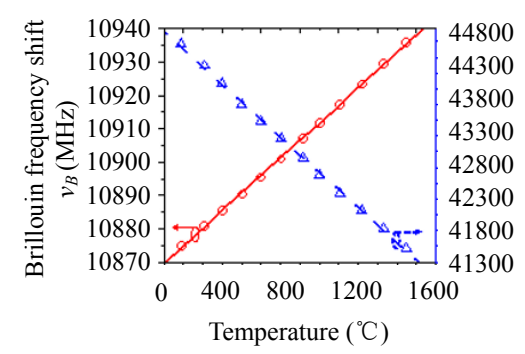

(b)

Fig. 8 Measured strain and temperature coefficients: (a) strain dependence and (b) temperature dependence [circles denote the experimental results for the Brillouin frequency shift $\left(v_{B}\right)$ in left vertical axes, and triangles correspond to the birefringence-determined frequency deviation $\left(f_{y x}\right)$ in right vertical axes, respectively] [26] (copyright@OSA OE). two quantities shift in the same way with the applied strain but in the opposite directions with the temperature. All dependence shows the excellent linearity; thus by linear fitting, we get the strain coefficient and the temperature coefficient of $C_{v}^{\mathcal{E}}=$ $+0.03938 \mathrm{MHz} / \mu \varepsilon$ and $C_{v}^{T}=+1.0580 \mathrm{MHz} /{ }^{\circ} \mathrm{C}$ for the BFS $v_{B}$ and $C_{f}^{\varepsilon}=+0.8995 \mathrm{MHz} / \mu \varepsilon$ and $C_{f}^{T}=$ $-55.8134 \mathrm{MHz} /{ }^{\circ} \mathrm{C}$ for the frequency deviation $f_{y x}$, respectively. Using our measured result $f_{y x 0}=$ 43.875 GHz [see Fig. 7(b)] together with Chiang et al.'s result [27] of $\left(\gamma_{3}-\gamma_{2}\right) /\left[\left(\alpha_{3}-\alpha_{2}\right)\left(T_{\text {fic }}-25\right)\right]=$ $24.3 \times 10^{-6} \mu \varepsilon^{-1}$, the coefficients in the fiber are deduced approximately to be $C_{f}^{\varepsilon}=+1.069 \mathrm{MHz} / \mu \varepsilon$ and $C_{f}^{T}=-54.8 \mathrm{MHz} /{ }^{\circ} \mathrm{C}$ according to (8) and (9), respectively. It means that the measured coefficients for the frequency deviation $f_{y x}$ match well with our theoretical estimations.

Putting above strain/temperature coefficients into (10) and taking the standard errors of our measurement system $\left(\delta v_{B}=0.1 \mathrm{MHz}\right.$ and $\delta f_{y x}$ $=4 \mathrm{MHz}$, respectively) into account, the accuracy of the discrimination is given as high as $\delta \varepsilon= \pm 3.1 \mu \varepsilon$ and $\delta T= \pm 0.078^{\circ} \mathrm{C}$. This high accuracy proves the ability of completely discriminative sensing of the strain and temperature. When a set of strain and temperature $(\Delta \varepsilon, \Delta T)$ was initialized as $\left(0 \mu \varepsilon, 4.9^{\circ} \mathrm{C}\right)$ or $\left(939 \mu \varepsilon, 0{ }^{\circ} \mathrm{C}\right)$ in Fig. 5, for example, two frequency changes $\left(\Delta v_{B}\right.$ and $\left.\Delta f_{y x}\right)$ were measured as $(5.1 \mathrm{MHz},-276 \mathrm{MHz})$ or (37.1 MHz, $849 \mathrm{MHz}$ ). According to (3), the strain and the temperature values are calculated as $(-2.3 \mu \varepsilon$, $\left.4.907{ }^{\circ} \mathrm{C}\right)$ or $\left(942.6 \mu \varepsilon,-0.0197{ }^{\circ} \mathrm{C}\right)$, respectively. They differ from the initialized values with errors as low as $\left(-2.3 \mu \varepsilon, 0.007{ }^{\circ} \mathrm{C}\right)$ or $\left(3.6 \mu \varepsilon,-0.020{ }^{\circ} \mathrm{C}\right)$. This example also verifies the complete discrimination ability of the proposed method.

\section{Distributed discrimination of strain and temperature}

\subsection{Proof-of-concept}

For distributed discrimination of strain and 
temperature, the localized BDG generation and readout in the PMF should be firstly proved to be effective. A correlation-based continuous-wave technique of the BOCDA system was used for random access [29], and a pulse-based time-domain technique was also employed for continuous access [30]. It was found that the generation and readout optical waves in the BOCDA system should be synchronously frequency-modulated because of the dispersion properties of all four waves (see Fig. 9) [31], including writing light waves (pump and probe), reading light wave (readout), and acoustic wave (dynamic grating as well). Consequently, the local BGS and BDG diffraction grating could be correctly identified, as schematically shown in Fig. 10 [29].

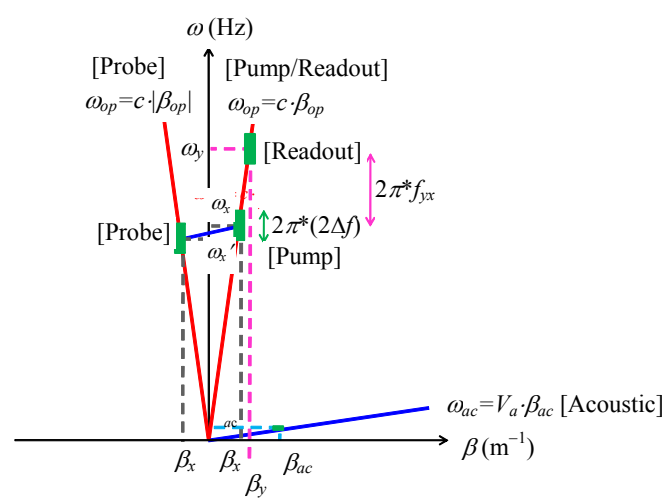

Fig. 9 Dispersion properties of all pump, probe and readout waves together with the acoustic wave (Brillouin dynamic grating) [31] (copyright@OSA OE).

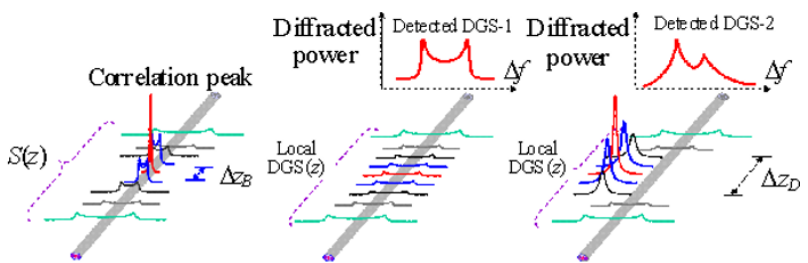

(a)

(b)

(c)

Fig. 10 Schematic of distributed measurement of DGS: (a) beat power spectrum distribution $S(z)$ near the correlation peak: the local dynamic grating spectrum (DGS) when the optical frequency of the readout wave is not modulated (b) or modulated synchronously to the modulation to pump light (c): the top plots show the measured DGS for (b) or for (c) when the correlation peak is localized in a heated segment [29] (copyright@OSA OL).

$$
d_{m}=\frac{c}{2 n_{\text {eff }} f_{m}}
$$

where $f_{m}$ is the modulation frequency of the lasers. However, their spatial resolutions are separately determined by the BGS linewidth $\left(\Delta v_{B}\right)$ and the BDG bandwidth $\left(\Delta f_{y x}\right)$ as follows [29]:

$$
\begin{aligned}
& \Delta z_{B}=\frac{c}{2 n_{\text {eff }} f_{m}} \frac{\Delta v_{B}}{\pi \Delta f} \\
& \Delta z_{D}=\frac{c}{2 n_{\text {eff }} f_{m}} \frac{\Delta f_{y x}}{\pi \Delta f}
\end{aligned}
$$

where $\Delta f$ is the modulation amplitude of the lasers.

Figure 11 depicts the experimental configuration of the distributed generation and readout of the BGS and BDG based on the correlation-based continuous-wave technique [29]. The difference from Fig. 6 is the introduction of the synchronous frequency modulation to the lasers. The measurement range of the distributed BGS and BDG is commonly given by the neighboring correlation peaks of the BOCDA system $[1,29]$.

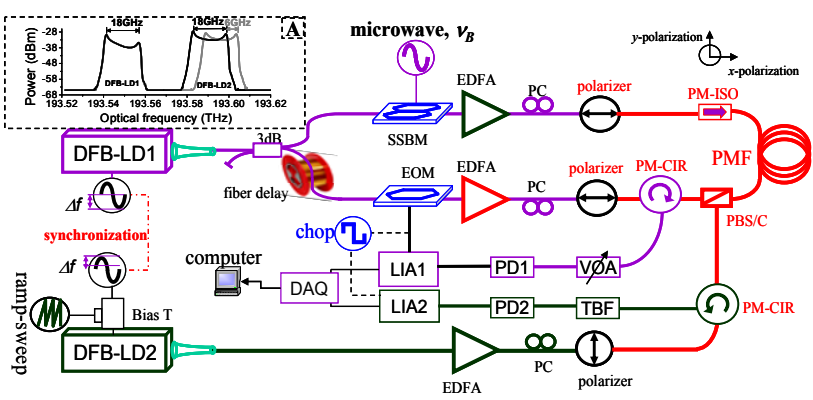

Fig. 11 Experimental configuration for the distributed generating and measuring the BGS and DGS in a PMF (copyright@OSA OL).

The proof-of-concept distributed measurement ability with the $1.2-\mathrm{m}$ spatial resolution was verified as demonstrated in Fig. 12. Four heated segments cascaded along the PMF sample were prepared [see Fig. 12(a)]. The measured BGS and BDG distribution in the first two segments are shown in Fig. 12(b); the characterized $v_{B}$ and $f_{y x}$ with opposite responses to the increased temperature are clearly illustrated in Fig. 12(c). 


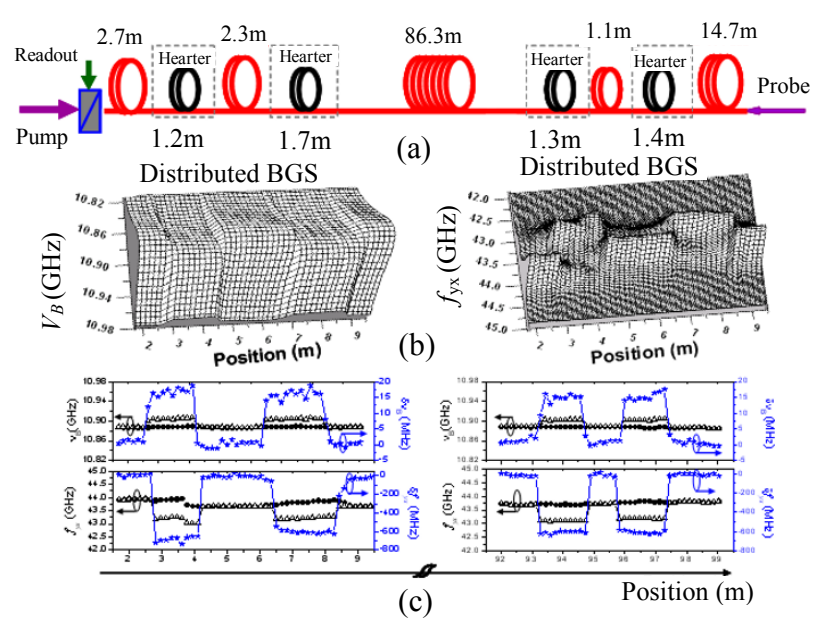

Fig. 12 Distributed measurement results: (a) prepared PMF sample, (b) examples of 3D distribution of measured BGS and DGS from $2 \mathrm{~m}$ to $9 \mathrm{~m}$ when the heater is turned on, and (c) summarization of the detected BFS $v_{B}$ (upper) or frequency deviation $f_{\mathrm{yx}}$ (lower) and their temperature-induced changes near the heated segments [29] (copyright@OSA OL).

\subsection{Two-laser-based scheme of distributed discrimination}

By use of the experimental setup shown in Fig. 11, the two-laser-based scheme of distributed discrimination of strain and temperature was successfully demonstrated with the $10-\mathrm{cm}$ spatial resolution [32]. The $f_{m}=12.429 \mathrm{MHz}$ determines the nominal measurement range as $d_{m}=8.35 \mathrm{~m}$ according to (11). For local BGS and BDG measurement, $\Delta f_{B}=1.5 \mathrm{GHz}$ and $\Delta f_{D}=10 \mathrm{GHz}$ correspond to the nominal spatial resolution $\Delta z_{B}=$ $5 \mathrm{~cm}$ and $\Delta z_{D}=8 \mathrm{~cm} \quad$ [see (12) and (13)], respectively.

To validate the feasibility of distributed discrimination of strain and temperature, we constructed an 8-m PMF sample consisting of nine (A-I) cascaded fiber portions of $10 \mathrm{~cm}-16 \mathrm{~cm}$ in length, which is illustrated in Fig. 13(a). The A, C, G, and I portions were loosely laid at $25.1{ }^{\circ} \mathrm{C}$ for reference, while the $\mathrm{B}, \mathrm{D}, \mathrm{F}$, and $\mathrm{H}$ portions were loosely inserted into a temperature-controlled water bath with the $0.1-{ }^{\circ} \mathrm{C}$ accuracy. The $\mathrm{E}$ portion was also inserted into the water bath and glued to a set of translation stages to load strain. The measured distribution of the changes in $\Delta v_{B}$ and $\Delta f_{y x}$ are summarized in Figs. 13(b) and 13(c), respectively. Referred to the characterized coefficients in Fig. 8 and the cross-sensitivity matrix in (10), the deducted distributions of strain and temperature along the fiber are depicted in Figs. 13(d) and 13(e), which clearly shows the feasibility of distributed discrimination of strain and temperature.

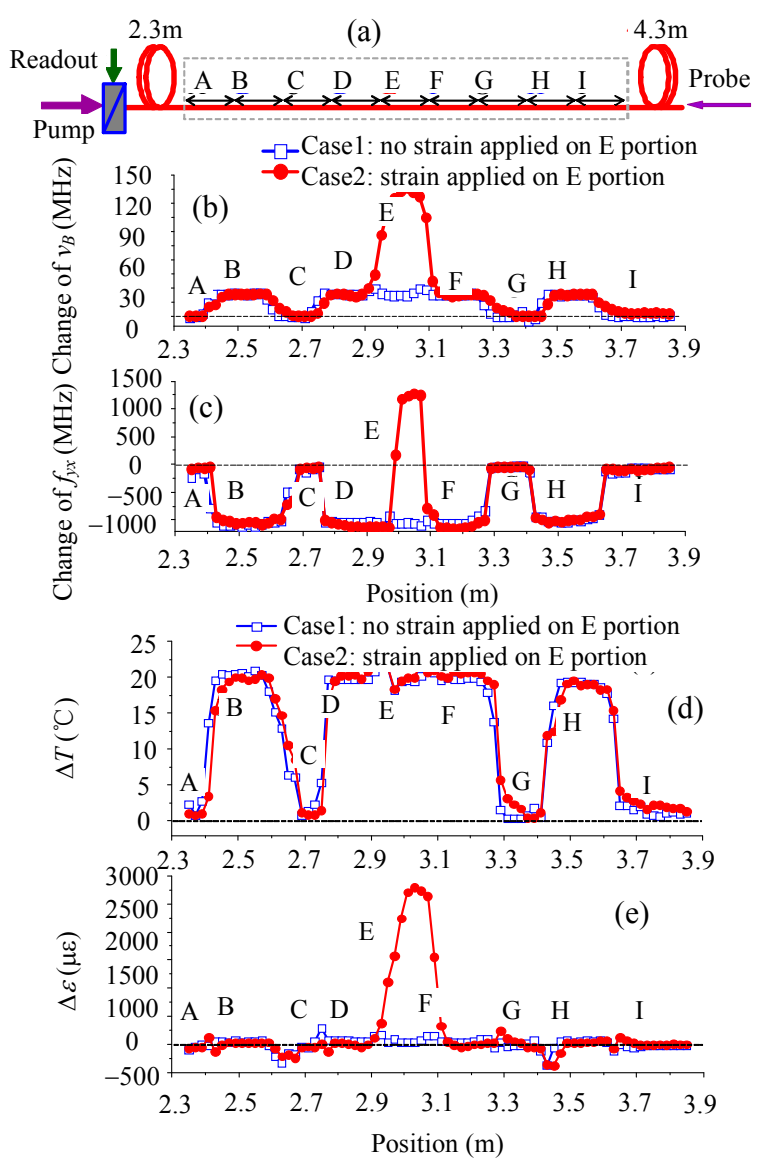

Fig. 13 PMF sample comprising 9 cascaded strained and heated fiber portions (dashed box) with $10 \mathrm{~cm}-16 \mathrm{~cm}$ in length (a), distribution of the change in the Brillouin frequency shift $v_{B}$ (b), the frequency deviation $f_{y x}$, and distributed discrimination of strain (d) and temperature (e) [32] (copyright@IEEE PTL).

\subsection{One-laser-based scheme of distributed discrimination}

Superior to all BDG generation/detection schemes using multiple individual laser sources [29, $30,32,33]$, the one-laser-based new scheme [31] can generate the coherent light waves to write the BDG (pump and probe waves) and to detect BDG 
(readout wave) by use of a sideband-generation technique. The new scheme overcomes the relative frequency fluctuations among multiple waves and ensures a much higher precision than previous schemes in the measured BGS and BDG. It can also provide a higher speed in the measurement of the BGS and BDG since the time-consuming averaging process is not necessary.

Figure 14 shows the experimental setup of one-laser-based generation and detection of the BDG in a PMF. A 1549-nm distributed-feedback laser diode (DFB-LD) serves as the laser source. A $40-\mathrm{GHz}$ intensity modulator (IM2) driven by a radio frequency synthesizer (RF2 at $\nu_{\mathrm{RF} 2}$ ) with a proper dc bias is used to generate double sidebands with the suppressed carrier (DSB-SC). The output of IM2 is launched into a fiber Bragg grating (FBG) through a circulator. The light reflected from the FBG, after further filtered by a tunable band-pass filter (TBF1), is used as the light source for pump and probe waves to write the BDG. After amplified by an erbiumdoped fiber amplifier (EDFA1), the light is divided into the pump and probe waves. The frequency of the probe wave is down-shifted by a single-sideband modulator (SSBM) driven by another synthesizer (RF1 at $v_{\mathrm{RF} 1}$ ); the pump is chopped by IM1 for lock-in detection. The pump and probe waves are linearly polarized along the $x$-axis (slow-axis) of the PMF, which is used as the sensing medium. On the other hand, the light passing through the FBG is used as the readout wave to interrogate the BDG. The readout wave is $y$-polarized and launched into the PMF in the same direction as the pump wave.

As shown in Fig. 15, the $f_{y x}$ measured in this one-laser-based scheme shows high stability and accuracy (several MHz), while that obtained by the two-laser scheme suffers very high fluctuation (several hundreds of $\mathrm{MHz}$ ). Compared to the previous two-laser scheme (see Fig. 11), the one-laser-based scheme demonstrated here has the similar spatial resolution $(\sim 10 \mathrm{~cm})$ and measurement range $(\sim 5 \mathrm{~m})$, but has several advantages including the faster measurement speed without time-consuming averaging, simpler measurement without sophisticated synchronization, and higher accuracy.

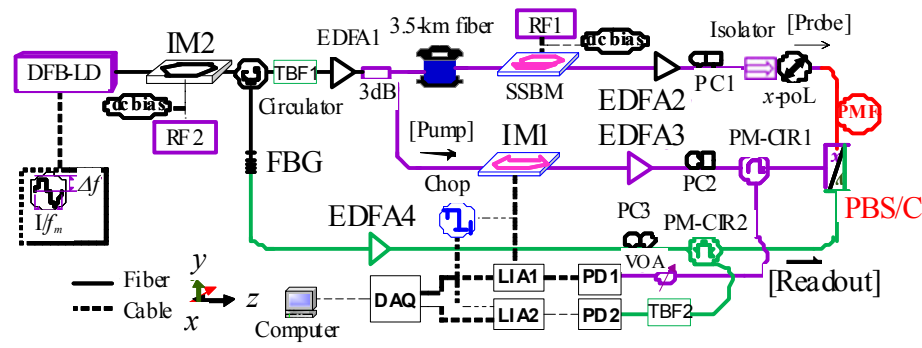

Fig. 14 Experimental setup of one-laser-based Brillouin dynamic grating generation/detection and discrimination of strain and temperature based on BOCDA (the light from DFB-LD is intensity-modulated to generate two sidebands with suppressed carrier) [31] (copyright@OSA OE).

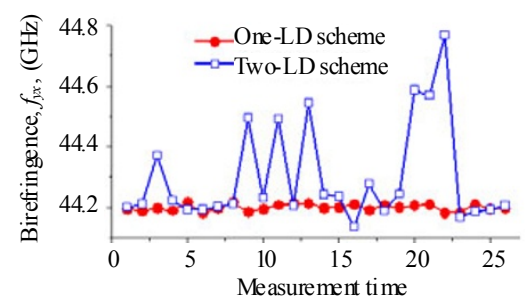

Fig. 15 Comparison of the BDG measurement accuracy between the one-laser scheme and two-laser scheme [31] (copyright@OSA OE).
Figure 16 summarizes the experimental results when the fiber is heated from $25^{\circ} \mathrm{C}$ to $30^{\circ} \mathrm{C}$ or/and the strain $(\varepsilon=2000 \mu \varepsilon)$ is applied both at the location of $3.1 \mathrm{~m}$. It is clear that the imposed strain increases both the $v_{B}$ and $f_{y x}$, while the heating has a contribution to the $f_{y x}$ opposite to that to the $v_{B}$. By referring to the strain and temperature coefficients of the $v_{B}$ and $f_{y x}$ [see (10)], we evaluated the distribution of the temperature and strain along the 
fiber. The results summarized in Figs. 16(c) and 16(d) match well with the setting situation.

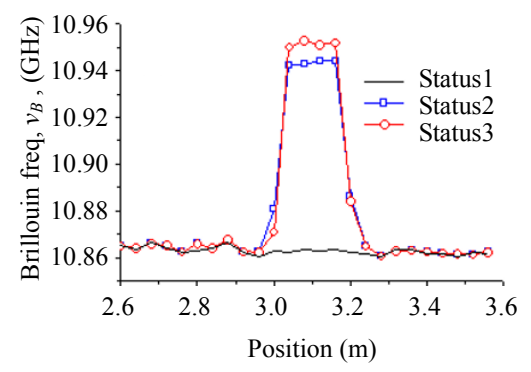

(a)

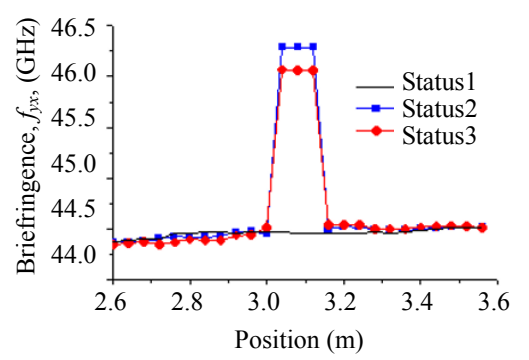

(b)

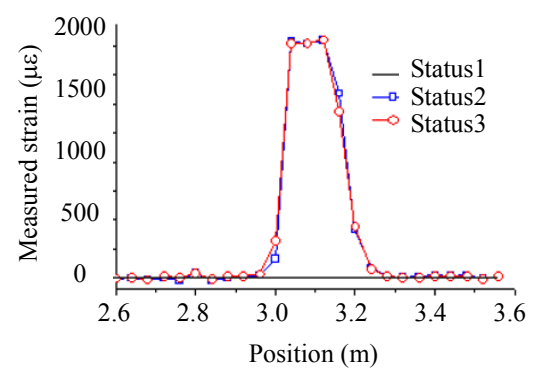

(c)

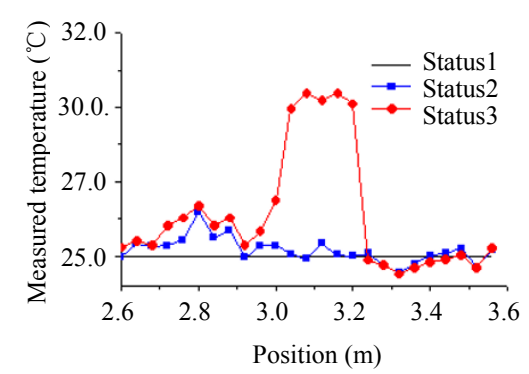

(d)

Fig. 16 Measured distribution of Brillouin frequency $v_{B}$ (a) and birefringence-determined frequency deviation $f_{y x}$ (b), discriminated distribution of strain (c) and temperature (d): solid lines (Status 1): $T=25^{\circ} \mathrm{C}, \varepsilon=0 \mu \varepsilon$; squares (Status 2): $T=25^{\circ} \mathrm{C}$, $\varepsilon=2000 \mu \varepsilon$; circles (Status 3): $T=30{ }^{\circ} \mathrm{C}, \varepsilon=2000 \mu \varepsilon$ [31] (copyright@OSA OE).

\subsection{Range elongation of one-laser-based scheme}

Due to the nature of the correlation-domain continuous-wave technique in the BOCDA system, the spatial resolutions of the distributed BGS and BDG measurement are in trade-off relation with the measurement range [see (11)-(13)]. It results in a limited range of the distributed discrimination of strain and temperature. To overcome it, the temporal gating [34] or the dual modulation method [35] is introduced into the one-laser-based discriminative sensing system.

The temporal gating method can be easily implemented by use of a pulse modulation of the RF2 that drives the IM2 in Fig. 14. The pulse modulation has $1 / N$ duty ratio and $1 / N$ repetition frequency compared to that of the LD frequency modulation. The pump-probe interaction occurs only at the positions where the counter propagating probe and pump pulses overlap [36]. As a result, the range given in (11) can be elongated by $N$ times. In the experiment, the spatial resolution was set at $50 \mathrm{~cm}$; the range increased by 20 times (from $25 \mathrm{~m}$ to $500 \mathrm{~m}$ ) [36].

A fiber under test of a 500-m-length fiber composed of two types of the PMF (A and B) is shown in Fig. 17(a). The fiber is emerged in a
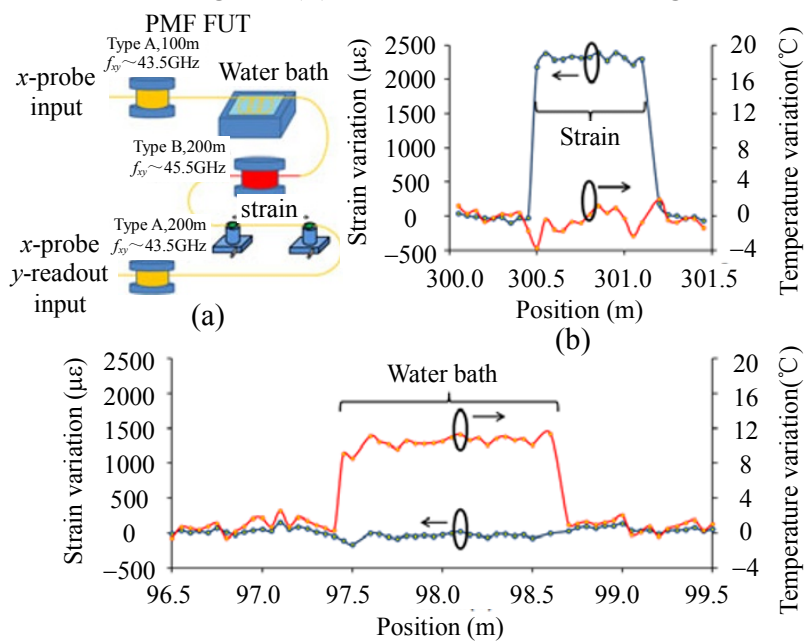

(c)

Fig. 17 Experimental results: (a) configuration of the fiber under test, and discriminative distributed measurements of strain (b) and temperature (c) [35] (copyright@IEEE PTL). 
temperature controlled water bath for temperature control or pulled with a movable stage to load strain. The distributed discrimination of strain and temperature along the fiber is illustrated in Figs. 17(b) and 17(c), which clearly show the feasibility of the elongated measurement range.

\section{Conclusions}

We have demonstrated the recent research progress on the discrimination of strain and temperature by use of a single-length optical fiber. After a brief introduction of the preliminary method based on a specially-designed optical fiber, the novel method based on the BDG in a PMF for complete discrimination of strain and temperature is presented. The fundamental principles as well as two different schemes of distributed discrimination of strain and temperature are overviewed. Thanks to the inherent coherence of the writing and reading waves of the BDG, the one-laser-based scheme has more features of the high speed and high accuracy, which are also easily extended with the assistance of the temporal gating method or the dual modulation method. The next research efforts will focus on the improvement in the sensing speed along the entire fiber [37, 38] and the simplification of the entire fiber-optic nerve system towards the real industry application in the smart materials and smart structure.

\section{Acknowledgment}

This work is supported by the "Grant-in-Aid for Scientific Research (S)" and the "Global Center of Excellence Program (G-COE)" from the Ministry of Education, Culture, Sports, Science and Technology, Japan. Weiwen Zou is partially supported by the National Natural Science Foundation of China (Grant No. 61007052), the International Cooperation Project from the Ministry of Science and Technology of China (Grant No. 2011FDA11780), Shanghai Pujiang Program from STCSM (Grant No. 12PJ1405600), and the "SMC Young Star" Scientist Program of Shanghai Jiao Tong University. Zuyuan
He is partially supported by the National Natural Science Foundation of China (Grant No. 61275097).

Open Access This article is distributed under the terms of the Creative Commons Attribution License which permits any use, distribution, and reproduction in any medium, provided the original author(s) and source are credited.

\section{References}

[1] K. Hotate and T. Hasegawa, "Measurement of Brillouin gain spectrum distribution along an optical fiber using a correlation-based technique - proposal, experiment and simulation," IEICE Transactions on Electronics, vol. E83-C, no. 3, pp. 405-412, 2000.

[2] K. Hotate and M. Tanaka, "Correlation-based continuous-wave technique for optical fiber distributed strain measurement using Brillouin scattering with cm-order spatial resolution applications to smart materials," IEICE Transactions on Electronics, vol. E84-C, no. 12, pp. 1823-1828, 2001.

[3] K. Y. Song, Z. He, and K. Hotate, "Distributed strain measurement with millimeter-order spatial resolution based on Brillouin optical correlation domain analysis," Optics Letters, vol. 31, no. 17, pp. 2526-2528, 2006.

[4] Y. Mizuno, W. Zou, Z. He, and K. Hotate, "Proposal of Brillouin optical correlation-domain reflectometry (BOCDR)," Optics Express, vol. 16, no. 16, pp. 12148-12153, 2008.

[5] Y. Mizuno, W. Zou, Z. He, and K. Hotate, "Operation of Brillouin optical correlation-domain reflectometry: theoretical analysis and experimental validation," Journal of Lightwave Technology, vol. 28, no. 22, pp. 3300-3306, 2010.

[6] Y. Mizuno, Z. He, and K. Hotate, "One-end-access high-speed distributed strain measurement with 13-mm spatial resolution based on Brillouin optical correlation-domain reflectometry," IEEE Photonics Technology Letters, vol. 21, no. 7, pp. 474-476, 2009.

[7] X. Bao, D. J. Webb, and D. A. Jackson, "32-km distributed temperature sensor based on Brillouin loss in an optical fiber," Optics Letters, vol. 18, no. 18, pp. 1561-1563, 1993.

[8] M. Nikles, L. Thevenaz, and P. Robert, "Simple distributed fiber sensor based on Brillouin gain spectrum analysis," Optics Letters, vol. 21, no. 10, pp. 758-760, 1996.

[9] T. Kurashima, T. Horiguchi, H. Izumita, S. Furukawa, and Y. Koyamada, "Brillouin optical-fiber time 
domain reflectometry," IEICE Transactions on Electronics, vol. E76-B, no. 4, pp. 382-390, 1993.

[10] M. N. Alahbabi, Y. T. Cho, and T. P. Newson, "150-km-range distributed temperature sensor based on coherent detection of spontaneous Brillouin backscatter and in-line Raman amplification," Journal of the Optical Society of America B, vol. 22, no. 6, pp. 1321-1324, 2005.

[11] T. Horiguchi, T. Kurashima, and M. Tateda, "Tensile strain dependence of Brillouin frequency shift in silica optical fibers," IEEE Photonics Technology Letters, vol. 1, no. 5, pp. 107-108, 1989.

[12] T. Kurashima, T. Horiguchi, and M. Tateda, "Thermal effects of Brillouin gain spectra in silica optical fibers," IEEE Photonics Technology Letters, vol. 2, no. 10, 718-720, 1990.

[13] W. Zou, Z. He, and K. Hotate, "Investigation of strain- and temperature-dependences of Brillouin frequency shifts in $\mathrm{GeO}_{2}$-doped optical fibers," Journal of Lightwave Technology, vol. 26, no. 13, pp. 1854-1861, 2008.

[14] G. P. Agrawal, Nonlinear fiber optics (3rd), New York: Academic Press, 2001.

[15] C. C. Lee, P. W. Chiang, and S. Chi, "Utilization of a dispersion-shifted fiber for simultaneous measurement of distributed strain and temperature through Brillouin frequency shift," IEEE Photonics Technology Letters, vol. 13, no. 10, pp. 1094-1096, 2001.

[16] L. Zou, X. Bao, and L. Chen, "Brillouin scattering spectrum in photonic crystal fiber with a partially germanium-doped core," Optics Letters, vol. 28, no. 21, pp. 2022-2024, 2003.

[17] W. Zou, Z. He, and K. Hotate, "Stimulated Brillouin scattering and its dependences on strain and temperature in a high-delta optical fiber with F-doped depressed inner cladding," Optics Letters, vol. 32, no. 6, pp. 600-602, 2007.

[18] W. Zou, Z. He, and K. Hotate, "Two-dimensional finite element modal analysis of Brillouin gain spectra in optical fibers," IEEE Photonics Technology Letters, vol. 18, no. 23, pp. 2487-2489, 2006.

[19] W. Zou, Z. He, and K. Hotate, "Acoustic modal analysis and control in w-shaped triple-layer optical fibers with highly-germanium-doped core and F-doped inner cladding," Optics Express, vol. 16, no. 14, pp. 10006-10017, 2008.

[20] W. Zou, Z. He, and K. Hotate, "Experimental study of Brillouin scattering in fluorine-doped single-mode optical fibers," Optics Express, vol. 16, no. 23, pp. 18804-18812, 2008.

[21] W. Zou, Z. He, and K. Hotate, "Dependence of Brillouin frequency shift in optical fibers on draw-induced residual elastic and inelastic strains," IEEE Photonics Technology Letters, vol. 19, no. 18, pp. 1389-1391, 2007.
[22] T. P. Parker, M. Farhadiroushan, V. A. Handerek, and A. J. Rogers, "Temperature and strain dependence of the power level and frequency of spontaneous Brillouin scattering in optical fibers," Optics Letters, vol. 22, no. 11, pp. 787-789, 1997.

[23] T. Okoshi, "Single-polarization single-mode optical fibers," IEEE Photonics Technology Letters, vol. 17, no. 6, pp. 879-884, 1981.

[24] K. Hotate, K. Makino, Z. He, M. Ishikawa, and Y. Yoshikuni, "High spatial resolution fiber-optic distributed lateral-stress sensing by stepwise frequency modulation of a super structure grating distributed Bragg reflector laser diode," IEEE Journal of Quantum Electronics, vol. 24, no. 7, pp. 2733-2740, 2006.

[25] K. Y. Song, W. Zou, Z. He, and K. Hotate, "All-optical dynamic grating generation based on Brillouin scattering in polarization-maintaining fiber," Optics Letters, vol. 33, no. 9, pp. 926-928 2008.

[26] W. Zou, Z. He, and K. Hotate, "Complete discrimination of strain and temperature using Brillouin frequency shift and birefringence in a polarization-maintaining fiber," Optics Express, vol. 17, no. 3, pp. 1248-1255, 2009.

[27] K. S. Chiang, D. Wong, and P. L. Chu, "Strain-induced birefringence in a highly birefringent optical fiber," Electronics Letters, vol. 26, no. 17, pp. 1344-1346, 1990.

[28] X. Bao, Q. Yu, and L. Chen, "Simultaneous strain and temperature measurements with polarization maintaining fibers and their error analysis by use of a distributed Brillouin loss system," Optics Letters, vol. 29, no. 12, pp. 1342-1344, 2004.

[29] W. Zou, Z. He, K. Y. Song, and K. Hotate, "Correlation-based distributed measurement of a dynamic grating spectrum generated in stimulated Brillouin scattering in a polarization-maintaining optical fiber," Optics Letters, vol. 34, no. 7, pp. 1126-1128, 2009.

[30] K. Y. Song, W. Zou, Z. He, and K. Hotate, "Optical time-domain measurement of Brillouin dynamic grating spectrum in a polarization maintaining fiber," Optics Letters, vol. 34, no. 9, pp. 1381-1383, 2009.

[31] W. Zou, Z. He, and K. Hotate, "One-laser-based generation/detection of Brillouin dynamic grating and its application to distributed discrimination of strain and temperature," Optics Express, vol. 19, no. 3, pp. 2363-2370, 2011.

[32] W. Zou, Z. He, and K. Hotate, "Demonstration of Brillouin distributed discrimination of strain and temperature using a polarization-maintaining optical fiber," IEEE Photonics Technology Letters, vol. 22, no. 8, pp. 526-528, 2010.

[33] Y. Dong, L. Chen, and X. Bao, "High-spatial-resolution time-domain simultaneous 
strain and temperature sensor using Brillouin scattering and birefringence in a polarization-maintaining fiber," IEEE Photonics Technology Letters, vol. 22, no. 18, pp. 1364-1366, 2010.

[34] R. K. Yamashita, W. Zou, Z. He, and K. Hotate, "Measurement range elongation based on temporal gating in Brillouin optical correlation domain distributed simultaneous sensing of strain and temperature," IEEE Photonics Technology Letters, vol. 24, no. 12, pp. 1006-1008, 2012.

[35] W. Zou, Z. He, and K. Hotate, "Enlargement of measurement range by double frequency modulations in one-laser Brillouin correlation-domain distributed discrimination system," presented at Conference on Lasers and Electro-Optics (CLEO) (Optical Society of America, 2011), Baltimore, paper CThL5, Baltimore,
Maryland, United States, May 1-6, 2011.

[36] M. Mure, M. Imai, and S. Miura, "Measurement range enlargement of Brillouin optical correlation domain analysis by pulse correlation method," in Proc. 42nd Meeting on Lightwave Technology, pp. 129-135, 2008.

[37] W. Zou, Z. He, and K. Hotate, "Realization of high-speed distributed sensing based on Brillouin optical correlation domain analysis," in Conference on Lasers and Electro-Optics (CLEO) (Optical Society of America, 2009), Baltimore, Maryland, United States, Jun. 2-4, pp. 1-2, 2009.

[38] W. Zou, Z. He, and K. Hotate, "Distributed dynamic-strain sensing based on Brillouin optical correlation domain analysis," in The 8th Pacific Rim Conference on Lasers and Electro-Optics (CLEO/PR) (Optical Society of America, 2009), Shanghai, China, Aug. 30-Sep. 3, pp. 1-2, 2009. 